\title{
Discrete Survival Model Analysis of Plasmodium falciparum Response to Artemisinin-Based Combination Therapies among Children in Regions of Varying Malaria Transmission in Cameroon
}

\author{
Akindeh M. Nji 1,2,3, Innocent M. Ali ${ }^{1,4}{ }^{(\mathbb{D}}$, Peter Thelma Ngwa Niba ${ }^{1,2}$ (D), Evehe Marie-Solange ${ }^{1,2}$, \\ Christian Heumann ${ }^{5}$ (D), Guenter Froeschl ${ }^{3,6}$ and Wilfred F. Mbacham ${ }^{1,2, *}$ \\ check for \\ updates \\ Citation: Nji, A.M.; Ali, I.M \\ Niba, P.T.N.; Marie-Solange, E.; \\ 1 The Biotechnology Center, University of Yaoundé I, Yaoundé BP 8094, Cameroon; \\ akindeh@yahoo.com (A.M.N.); dr.alinn@gmail.com (I.M.A.); thelma2009@yahoo.co.uk (P.T.N.N.); \\ seveheb@yahoo.com (E.M.-S.) \\ 2 Department of Biochemistry, University of Yaoundé I, Yaoundé BP 8094, Cameroon \\ 3 Center for International Health, Ludwig Maximilian University of Munich, 80802 Munich, Germany; \\ froeschl@lrz.uni-muenchen.de \\ 4 Department of Biochemistry, University of Dschang, Dschang BP 67, Cameroon \\ 5 Department of Statistics, Ludwig Maximilian University of Munich, 80802 Munich, Germany; \\ chris@stat.uni-muenchen.de \\ 6 Division of Infectious Diseases and Tropical Medicine, University Hospital, Ludwig-Maximilians-Universität, \\ 80802 Munich, Germany \\ * Correspondence: wfmbacham@yahoo.com
} Heumann, C.; Froeschl, G.; Mbacham, W.F. Discrete Survival Model Analysis of Plasmodium falciparum Response to Artemisinin-Based Combination Therapies among Children in Regions of Varying Malaria Transmission in Cameroon. Pathogens 2021, 10, 1106. https://doi.org/10.3390/pathogens 10091106

Academic Editor:

Andrew Taylor-Robinson

Received: 9 July 2021

Accepted: 27 August 2021

Published: 30 August 2021

Publisher's Note: MDPI stays neutral with regard to jurisdictional claims in published maps and institutional affiliations.

Copyright: (c) 2021 by the authors. Licensee MDPI, Basel, Switzerland. This article is an open access article distributed under the terms and conditions of the Creative Commons Attribution (CC BY) license (https:/ / creativecommons.org/licenses/by/ $4.0 /)$.

\begin{abstract}
The need to monitor changes in parasite clearance following treatment with artemisininbased combination therapies (ACTs) is important in the containment of drug resistance. This study aimed to model Plasmodium falciparum response to ACTs among children in two different transmission settings (Mutengene and Garoua) in Cameroon. Using the step function, a discrete-time survival model was fitted with all the covariates included that might play a role in parasite clearance. The probability of clearing parasites within $24 \mathrm{~h}$ following treatment was $21.6 \%$ and $70.3 \%$ for younger children aged 6 to 59 months and $29.3 \%$ and $59.8 \%$ for older children aged 60 to 120 months in Mutengene and Garoua, respectively. After two days of treatment, the conditional probability of clearing parasites given that they were not cleared on day 1 was $76.7 \%$ and $96.6 \%$ for children aged 6-59 months and $83.1 \%$ and $93.5 \%$ for children aged 60-120 months in Mutengene and Garoua, respectively. The model demonstrated that the ecological setting, age group and pretreatment serum levels of creatinine and alanine aminotransferase were the main factors that significantly influenced parasite clearance in vivo after administration of ACTs $(p<0.05)$. The findings highlight the need for further investigations on host differential response to ACTs in current practice.
\end{abstract}

Keywords: Plasmodium falciparum; parasite clearance; children; ACT; discrete time; survival model; Cameroon

\section{Introduction}

The introduction of highly efficacious artemisinin-based combination treatments (ACTs) as first-line treatment in most malaria-endemic countries has recently contributed to notable reductions in childhood morbidity and mortality across sub-Saharan Africa [1,2]. However, clinical and molecular studies suggest de novo emergence of artemisinin-resistant Plasmodium falciparum parasites in the Thailand-Cambodia border area, where the standard ACT is artesunate-mefloquine [3,4]. The P. falciparum Kelch 13 (K13) propeller variants are the main markers responsible for resistance to artemisinins [5]. In Africa, even though resistance to the artemisinins is not yet a threat to malaria treatment, recent studies have reported the presence of $\mathrm{R} 561 \mathrm{H}$ and $\mathrm{P} 574 \mathrm{~L}$ polymorphisms conferring partial resistance to 
ACTs [6-9]. The human host factors responsible for delayed parasite clearance after the administration of ACTs have not been fully understood. By measuring parasite clearance rates in genetically identical and nonidentical parasites, some authors found that variations in parasite clearance following treatment with artemisinin derivatives were largely affected by parasite genetic elements [10]. Drug pressure in communities where self-medication is common has the potential of reducing parasite sensitivity to drugs, resulting in the selection of resistant clones [11,12]. Furthermore, factors such as endemicity and host immunity play a crucial role in defining the emergence and spread of drug resistance [13,14].

Using data from different studies, a few models have been fitted to describe patterns in antimalarial drug resistance and efficacy. Some of these models have described patterns in drug use, strategies to delay the progress of drug resistance [15], the role of antimalarial drugs in elimination of malaria [16] and the impact of ACTs and long-acting treatments in reducing malaria transmission [17]. Pongtavornpinyo and colleagues in 2008 described several factors that may affect ACT efficacy [18]. Treatment failure, coverage with ACTs, self-medication and presumptive treatment were identified as affecting resistance depending on the transmission level. This model measured the spread of drug resistance with the assumption that it already existed. Furthermore, Okell and colleagues in modeling intrahost parasite dynamics treated with ACTs demonstrated that parasite ring stage specificity to drug sensitivity was driving artemisinin resistance [17].

Although there are artemisinin resistance containment strategies spearheaded by the World Health Organization (WHO), combating this resistance will require many unknowns to be addressed [19]. Indeed, Maude et al. uncovered parasite clearance half-life as a strong predictor of the likelihood of artemisinin resistance individual patients and suggested that the "day 3" threshold of 10\% parasitemia reduction might be a relevant measure to predict the likelihood of resistance development [16]. However, this result is relevant in low transmission settings [16]. Therefore, in modeling the ACT response in humans treated for uncomplicated P. falciparum malaria, the incorporation of ecological and parasite factors has the potential to uncover other likely predictors of artemisinin resistance, especially in high transmission settings in Africa where the emergence of artemisinin resistance is yet to occur. The time to parasite clearance with respect to these factors could also be helpful in understanding the progress of artemisinin resistance.

\section{Materials and Methods}

\subsection{Study Design}

Data from a three-arm, open-labeled, randomized controlled noninferiority trial comparing the efficacy, safety and tolerability of artesunate-amodiaquine (AS-AQ) and dihydroartemisinin-piperaquine (DHA-PPQ) to artemether-lumefantrine (AL) in children aged 6 to 120 months during a 42-day follow-up period were used [20].

\subsection{Study Site and Setting}

Participants were enrolled from two ecological regions in Cameroon with different malaria transmission patterns. Mutengene, a malaria holoendemic junction town that is in the semi-mangrove and tropical wet forest region, has perennial transmission with seasonal spikes, while Garoua, which is in the Guinea Sahel-savannah belt, experiences seasonal malaria with marked spikes in transmission during the beginning of the rainy season in May-June.

\subsection{Study Population}

Participants in this study were P. falciparum malaria positive patients, either gender and 6 to 120 months of age visiting the hospital for care.

Participants were enrolled if (i) they were microscopically confirmed (using Giemsastained thick film) with uncomplicated P. falciparum malaria with 1000-100,000 asexual parasites $/ \mu \mathrm{L}$, (ii) they had fever with an axillary temperature $\geq 37.5^{\circ} \mathrm{C}$ or history of fever in the past $24 \mathrm{~h}$, (iii) they were able to ingest tablets orally and (iv) their parents or 
guardians gave consent on their behalf to take part in the study and visit the clinic on days of follow-up.

Participants were excluded if (i) they had mixed infection with other Plasmodium species, (ii) they were microscopically confirmed (using Giemsa-stained thick film) with uncomplicated P. falciparum malaria with $<1000$ or $>100,000$ asexual parasites $/ \mu \mathrm{L}$, (iii) they had an absence of fever or an axillary temperature $<37.5^{\circ} \mathrm{C}$, (iv) they had any danger signs of severe malaria, (v) they were unable to ingest tablets orally or (vi) their parents or guardians refused to give consent on their behalf to take part in the study and visit the clinic on days of follow-up.

\subsection{Study Procedure}

Giemsa-stained thick and thin blood smears were prepared from finger-pricked blood samples according to WHO guidelines to assess the presence and quantification of malaria parasites [21,22]. Primary inclusion into the study was based on a positive thick smear reading. Blood smears were examined under a microscope for determination of parasitemia on all visit days (days $0,1,2,3,7,14,21,28,35$ and 42 ) and for all unscheduled visits. Using the same clean microscope slide, approximately $5 \mu \mathrm{L}$ of capillary blood was used for thick smear preparation, and $2 \mu \mathrm{L}$ was used for thin smear preparation. The smears were allowed to air dry and the thin smears were fixed in absolute methanol before staining with freshly prepared 10\% Giemsa stain for 15-20 min [22]. The slides were washed with tap water and air-dried before examination using $100 \times$ objective light microscope [22]. Parasitemia was quantified by a standard approximation method $(40 \times$ the number of parasites per 200 leucocytes on thick film) [22]. Malaria parasite speciation and detection of gametocytes (asexual stages) were done using the thin blood films [22]. The gametocytes were reported as present or absent. Two trained microscopists observed the slides [22]. Quality control of the microscopy readings was done by mask reading of $10 \%$ of the slides at the Immunology Laboratory of the Biotechnology Center, University of Yaoundé I. The average parasitemia was calculated in cases with discordant readings of less than $50 \%$ between the first two readers. However, the expert microscopist was used when the readings had a disagreement of more than $50 \%$ between the first and second readers [22].

Hemoglobin levels were measured on days 0,7 and 42 and on any unscheduled visit using a HemoCue B-Hemoglobinometer (HemoCue, Ängelholm, Sweden). A full blood count, including differentials, and biochemical parameters of liver and kidney functions (alanine aminotransferase serum activity, bilirubin and creatinine serum concentrations), were investigated from venous blood before treatment and on days 7 and 42 or on the day of reappearance of parasitemia.

Polymerase chain reaction (PCR) analysis of the polymorphic antigen markers merozoite surface proteins ( $m s p-1, m s p-2)$ and glutamate-rich protein (glurp) of P. falciparum parasites was used to distinguish reinfections from recrudescences in all parasite recurrences during the period of follow-up. Malaria cases were classified as new infections when there were no common bands observed between day 0 and the day of recurrent parasitemia. However, a case was categorized as recrudescence when there was at least one common band between baseline sample and that of the day of parasite reappearance for any of the 3 markers (even if there were additional bands on day 0 ). Malaria parasite infected cases were considered not to be clinical failures if their recurrent parasitemia were classified as new infections rather than recrudescent infections. There were 720 children recruited in this study. The results of the randomized three-arm, open-label, noninferiority clinical trial were published elsewhere [20].

\subsection{Statistical Analysis}

Parasite density for each patient was determined in the morning on the fixed visit days. Therefore, only the interval of time when parasites were cleared for each patient was known. Hence, a discrete-time survival model was fitted instead of the conventional proportional hazard model which assumes time to event to be continuous and that the 
exact instance of parasite clearance is known. Any visit in which the patient's parasitemia was microscopically undetectable was considered an event. Those who had cleared their parasites before being lost to follow-up and those not lost to follow-up who did not clear their parasites were included in the data set. The covariates used in the model were as follows: site (Garoua or Mutengene), the administered drug (AL, DHA-PPQ or AS$\mathrm{AQ}$ ), the residence type (urban or rural), the sex (male or female), age group (6 to 59 months, 60 to 120 months), weight and pretreatment temperature. Possible interacting factors were simultaneously included in the model to assess their combinatorial effect on parasite clearance in vivo. The combinations included the following: age and drug, site and drug. Given that some liver and kidney function tests and hematological parameters were assessed only on days 0,7 and 42, only the pretreatment values were considered with the assumption that their correlation to parasite clearance would be most relevant before treatment. To this end, the following parameters were also included in the model: alanine aminotransferase (ALAT) levels (normal range: 3-61 U/L), creatinine levels (normal range: $0.5-1.4 \mathrm{mg} / \mathrm{dL}$ ), relative neutrophil levels (as per proportion of leucocyte count; normal range: $42-72 \%$ ) and hemoglobin levels (normal range: $\geq 10 \mathrm{~g} / \mathrm{dL}$ ). The liver and kidney function tests were dichotomized in the model. The acetylator status (slow acetylator and fast acetylator) was also included as a predictor in the model. The phenotype of drug metabolic status was determined using polymerase chain reaction-restrictive fragment length polymorphism (PCR-RFLP) of the phase II enzyme encoded by the Nacetyltransferase 2 (NAT2) gene. This approach was used as a proxy for the measurement of pharmacokinetics in the absence of blood drug concentration.

A discrete-time survival model was fitted with day of visit as the discrete time given that only the interval was recorded rather than using the precise hour during which the parasites would no longer be detectable microscopically. The time points were the days patients visited the clinic for clinical evaluation. The hazard function was obtained by reparameterizing these probabilities to have a logistic dependence on predictors and time. The discrete hazard model is given by:

$$
\mathrm{h}_{\mathrm{ij}}=\frac{1}{1+\exp \left(-\left[\left(\alpha_{1} \mathrm{D}_{1 \mathrm{ij}}+\alpha_{2} \mathrm{D}_{2 \mathrm{ij}}+\ldots+\alpha_{\mathrm{J}} \mathrm{D}_{\mathrm{Jij}}\right)+\left(\beta_{1} \mathrm{Z}_{1 \mathrm{ij}}+\beta_{2} \mathrm{z}_{2 \mathrm{ij}}+\ldots+\beta_{\mathrm{P}} \mathrm{Z}_{\mathrm{Pij}}\right)\right]\right)}
$$

where $h_{i j}$ is the probability that an individual $i$ experiences the event at time $j$ and $\left[D_{1 i j}, D_{2 i j}\right.$, $\left.\ldots, \mathrm{D}_{\mathrm{Jij}}\right]$ are a sequence of dummy variables with values $\left[\mathrm{d}_{(1) \mathrm{ij}}, \mathrm{d}_{(2) \mathrm{ij}}, \ldots, \mathrm{d}_{(\mathrm{J}) \mathrm{ij}}\right]$ indexing time periods. In this model, "J" refers to the day of clearance, $\left[\alpha_{1}, \alpha_{2}, \ldots, \alpha_{J}\right]$ represent the baseline level of hazard in each time period and the slopes $\left[\beta_{1}, \beta_{2}, \ldots, \beta_{p}\right]$ describe the effects of predictors on the baseline hazard function on a logistic scale. The following equation was obtained by taking the logit transformation of Equation (1) above [23]:

$$
\log _{e}\left(\frac{h_{i j}}{1-h_{i j}}\right)=\left(\alpha_{1} D_{1 i j}+\alpha_{2} D_{2 i j}+\ldots+\alpha_{J} D_{j i j}\right)+\left(\beta_{1} Z_{1 i j}+\beta_{2} z_{2 i j}+\ldots+\beta_{P} Z_{P i j}\right)
$$

Hazard functions transformed this way provided the conditional log odds that an event will occur in each time point (visit day) given that the individual did not experience the event in the previous time point (visit). This is expressed as a linear function of $\alpha_{j}$ specific to time $j$, with the values of the predictors at time $j$ multiplied by the appropriate slopes $\left(\beta_{\mathrm{j}}\right)$.

This model was fitted using " $\mathrm{glm}$ " function in $\mathrm{R}$ statistical software version 4.1.0 (supported by the $R$ Foundation for Statistical Computing, Vienna, Austria) with logit function as link function. To fit this model using logistic regression, the data were first converted to a person-time data set such that each participant had one record corresponding to the discrete observed time. Therefore, the number of records for each participant corresponded to the number of discrete observed times until the event was observed (Supplementary Table S1). 
The hazard conditional probabilities were assessed by plotting predicted probabilities per time point and per predictor. Based on the fitted conditional hazard probabilities, the survival probability at time $\mathrm{j}$ can be obtained by Equation (3):

$$
\hat{s}_{j}=\prod_{k=1}^{k}\left(1-\hat{h}_{k}\right)
$$

where $\hat{h}_{k}$ is the conditional probability at time $k$. The estimated values of $S_{j}$ where $j=1,2$, $\ldots, \mathrm{m}$ were used to plot the survival function.

The discrete-time model was fitted on the assumption that the linear-logistic model was a valid representation of reality (linearity) and that all heterogeneity across individuals was accounted for by the variation of the values of the covariates. It was also assumed that the logit-hazard profiles corresponding to all possible values of every predictor are distinguished only by their constant vertical separation (proportionality). Linearity assumption was confirmed by fitting a generalized additive model while smoothing all the continuous variables. The degrees of freedom (df) of the continuous variables close to 1 indicated that the linearity assumption was tenable. To investigate the assumption of no unobserved heterogeneity, a mixed model was fitted with each subject as a random effect. A very small variation accounted for by the random effect showed that all the covariates in our model covered almost all the variation in the data. The proportionality assumption was investigated by verifying that the logit-hazard profiles estimated separately within strata were all approximately parallel.

In all analyses, two-tailed $p$ values less than 0.05 at $95 \%$ confidence level were considered to be statistically significant.

\section{Results}

\subsection{Description of the Study Participants and Parameters}

In this study, participants were randomized to receive three ACTs at Mutengene $(\mathrm{AS}-\mathrm{AQ}=144, \mathrm{DHA}-\mathrm{PPQ}=144$ and $\mathrm{AL}=72)$ and Garoua $(\mathrm{AS}-\mathrm{AQ}=144, \mathrm{DHA}-\mathrm{PPQ}=144$ and $\mathrm{AL}=72)$, which are ecologically different. Out of 720 enrolled patients, $23(3.2 \%)$ dropped out after the first dose, $74(10.3 \%)$ were lost to follow-up prior to reaching parasite clearance and $623(86.5 \%)$ were used in the modeling phase. This study included the following parameters: site, drug type, age, ecotype, urbanicity, parasite density, weight, temperature, hemoglobin level, relative neutrophil level, drug metabolizing status, alanine aminotransferase level and creatinine level.

\subsection{Association between Study Sites and Parasite Density}

There was a difference in the geometric mean parasite count between the two sites (31,039 asexual parasites/ $\mu \mathrm{L}$ for Garoua and 33,479 asexual parasites/ $\mu \mathrm{L}$ for Mutengene). However, the mean log parasite counts for the two sites were not significantly different $(t=-1.576$, degree of freedom $(\mathrm{df})=613, p=0.11)$. The $95 \%$ confidence interval $(95 \% \mathrm{CI})$ of the log parasite count was -0.15 to 0.02 , indicating that between sites, there was no significant difference $(p>0.05)$ in pretreatment parasite density.

\subsection{Discrete-time Survival Model Diagnostics}

The degrees of freedom (df) greater than 1 in a generalized additive model involving smoothened continuous variables are indicative of a breakdown in the linearity assumption. Hence, a generalized additive model was fitted using the variables of the full model and smoothing the continuous variables (weight, pretreatment temperature) in the model. The variable "weight" did not show any challenge to the assumption of linearity (degree of freedom of 1.001). However, the variable "temperature" showed a deviation from the linearity assumption (degree of freedom of 3.077), which was not statistically significant $(p=0.16)$. Therefore, the linearity assumption of the predictors was tenable. 
Fitting a random intercept model to the final selected predictors, with subjects or individual patients as random effects, we found the variance captured by random effects to be $7.8 \times 10^{-7}$ (standard deviation of $2.8 \times 10^{-4}$ ). The magnitude of variance can be considered negligible. Therefore, the assumption of unobserved heterogeneity uncorrelated with the independent variables in the model was valid. Separate plots of logit hazard functions within each stratum were approximately parallel. Proportionality was therefore assumed. Hence, there is evidence that the assumptions for this discrete-time survival model were reasonable.

\subsection{Survival Model Fitting}

The model arrived at was a discrete-time survival model incorporating time and all variables suspected of influencing parasite clearance (Table 1 ). The model showed that significant parasite clearance rates occurred on days 1, 2, 3, 7 and $14(p<0.05)$. Similarly, the site (Mutengene) and normal serum ALAT level on day 0 demonstrated a significant effect on parasite clearance $(p<0.05)$. Conversely, normal pretreatment serum creatinine level was significantly associated with delay in parasite clearance $(p<0.05)$. However, the other predictors did not show any significant effect $(p>0.05)$. The interaction between age group and site was important in determining time to parasite clearance at a 5\% significance level.

Table 1. Discrete-time survival model parameter estimates with standard errors.

\begin{tabular}{|c|c|}
\hline Predictors & Model Parameters $\beta$ (Standard Error) \\
\hline Day 1 & $4.63(0.43) * * *$ \\
\hline Day 2 & $7.14(0.48)^{* * *}$ \\
\hline Day 3 & $6.75(0.65) * * *$ \\
\hline Day 7 & $5.04(0.63) * * *$ \\
\hline Day 14 & $6.60(1.16) * * *$ \\
\hline Day 21 & $-9.77(533.41)$ \\
\hline Sex (male) & $-0.10(0.15)$ \\
\hline Age group $>5$ years & $-0.76(0.40)$ \\
\hline Site (Mutengene) & $4.05(1.29) * *$ \\
\hline AS-AQ & $-0.14(0.36)$ \\
\hline DHA-PPQ & $-0.17(0.36)$ \\
\hline Fast metabolizer & $0.03(0.16)$ \\
\hline Normal neutrophils level day 0 (\%) & $-0.13(0.16)$ \\
\hline Normal ALAT level day 0 (U/L) & $0.46(0.22) *$ \\
\hline Ecotype (Forest) & $1.49(1.23)$ \\
\hline Ecotype (Sahel) & $-1.26(1.70)$ \\
\hline Urbanicity (Urban) & $-0.03(0.26)$ \\
\hline Weight $(\mathrm{kg})$ & $0.01(0.02)$ \\
\hline Temperature $\left({ }^{\circ} \mathrm{C}\right)$ & $-0.09(0.11)$ \\
\hline Hemoglobin level > $10 \mathrm{~g} / \mathrm{dL}$ & $0.26(0.16)$ \\
\hline Normal creatinine level at day $0(\mathrm{mg} / \mathrm{dL})$ & $-0.64(0.21)^{* *}$ \\
\hline Age group $>5$ years $\times$ AS-AQ & $0.12(0.41)$ \\
\hline Age group $>5$ years $\times$ DHA-PPQ & $-0.01(0.41)$ \\
\hline Site (Mutengene) $\times$ AS-AQ & $0.36(0.41)$ \\
\hline Site (Mutengene) $\times$ DHA-PPQ & $0.69(0.42)$ \\
\hline Age group $>5$ years $\times$ Site (Mutengene) & $1.00(0.31)^{* *}$ \\
\hline
\end{tabular}

The odds ratios of variables in the final model showed that those with normal pretreatment ALAT activity (normal (3-61 U/L)) had a higher probability of clearing their parasites earlier $(\mathrm{OR}=1.58,95 \% \mathrm{CI}$ : 1.15-2.01) when compared to those with abnormal levels. However, having normal creatinine (normal $(0.5-1.4 \mathrm{mg} / \mathrm{dL})$ ) levels was associated with delay in parasite clearance $(\mathrm{OR}=0.53,95 \% \mathrm{CI}$ : $0.12-0.67)$ compared to those having abnormal creatinine levels (less than the lower normal limit $-<0.5 \mathrm{mg} / \mathrm{dL}$ ). Parasite clearance appeared to be delayed in Mutengene $(\mathrm{OR}=0.12,95 \% \mathrm{CI}$ : $0.07-0.18)$ when compared to 
Garoua. In Mutengene, older children (60-120 months) had a higher probability ( $\mathrm{OR}=2.72$, 95\% CI: 2.11-3.33) than children aged 6-59 months of clearing their parasites (Table 2).

Table 2. Odds ratios and 95\% confidence interval for significant variables of the model.

\begin{tabular}{ccc}
\hline Predictors & Odd Ratio Estimate & 95\% Confidence Interval \\
\hline Site (Mutengene) & 0.12 & $(0.07,0.18)^{*}$ \\
Normal ALAT level day 0 & 1.58 & $(1.15,2.01)^{*}$ \\
Normal creatinine level at day 0 & 0.53 & $(0.12,0.67)^{*}$ \\
Age group > 5 years $\times$ Site (Mutengene) & 2.72 & $(2.11,3.33) *$ \\
\hline Legend: * significant predictors, hazard = clearing parasite, ALAT = alanine aminotransferase, normal ALAT \\
level = 3-61 U/L, normal creatinine level = 0.5-1.4 mg/dL.
\end{tabular}

The time effect on the hazard function (Figure 1A) showed that on day 2, there was a higher probability of children clearing their parasites if they had not already done so on day 1. By day 3, most children had cleared their parasites (Figure 1B).

Hazard conditional Probabilities irrespective of heterogeneity

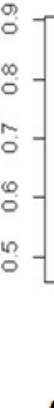

A

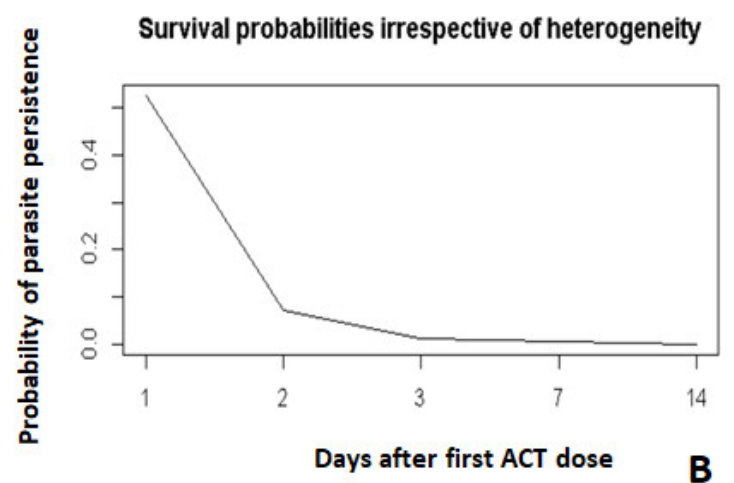

Figure 1. Hazard and survival probability profiles of the time effect on parasite clearance. Hazard conditional probabilities irrespective of heterogeneity (A). Survival probabilities irrespective of heterogeneity (B).

Day 0 was not included. It corresponds to the day when the first dose of ACT was administered.

Children in Mutengene lagged behind those in Garoua in parasite clearance, especially in the first two days after treatment (Figure 2A,B). Older children in Mutengene (60-120 months; day 1: $29.3 \%$, day 2: $83.1 \%$ ) had a better parasite clearance prognosis compared to the younger ones (6-59 months; day 1: $21.6 \%$, day 2: $76.6 \%$ ) (Figure 2A). In Garoua (Figure 2B), the opposite trend was observed.

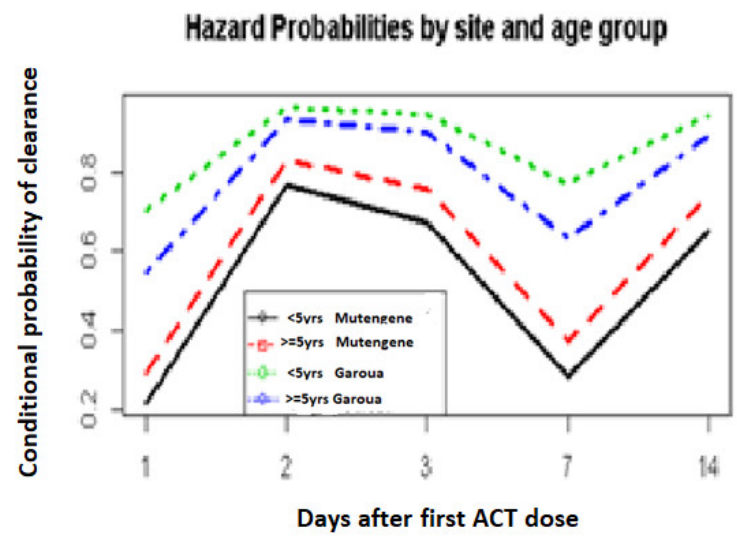

A

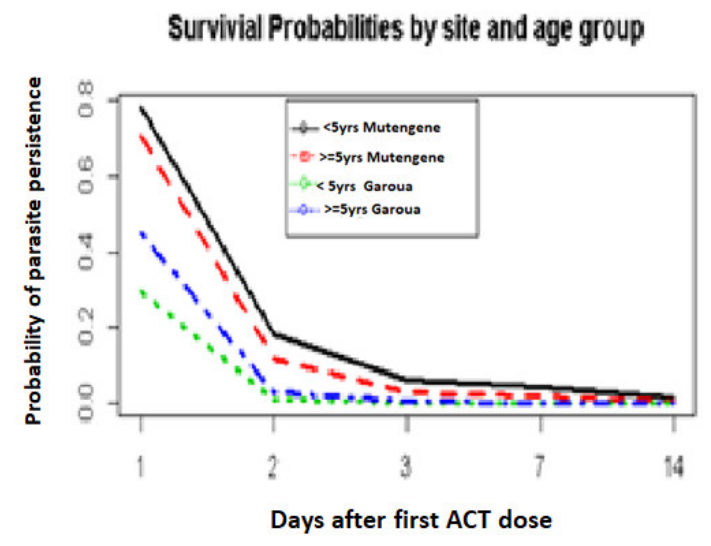

B

Figure 2. Hazard and survival probabilities by site and age group. Hazard probabilities by site and age group (A). Survivial probabilities by site and age group (B). 
Day 0 was not included. It corresponds to the day when the first dose of ACT was administered.

The ALAT levels in patients before treatment were correlated with time to parasite clearance (Figure 3A,B). Those with normal ALAT levels before treatment remained consistent with a higher chance of clearing their parasites for all the visit days on the condition that they did not clear the previous day of visit (Figure 3A). The probabilities of clearing parasites 2 days after treatment given that they were not cleared 1 day after treatment for normal and abnormal ALAT levels were $83 \%$ and $76 \%$, respectively (Figure 3A).
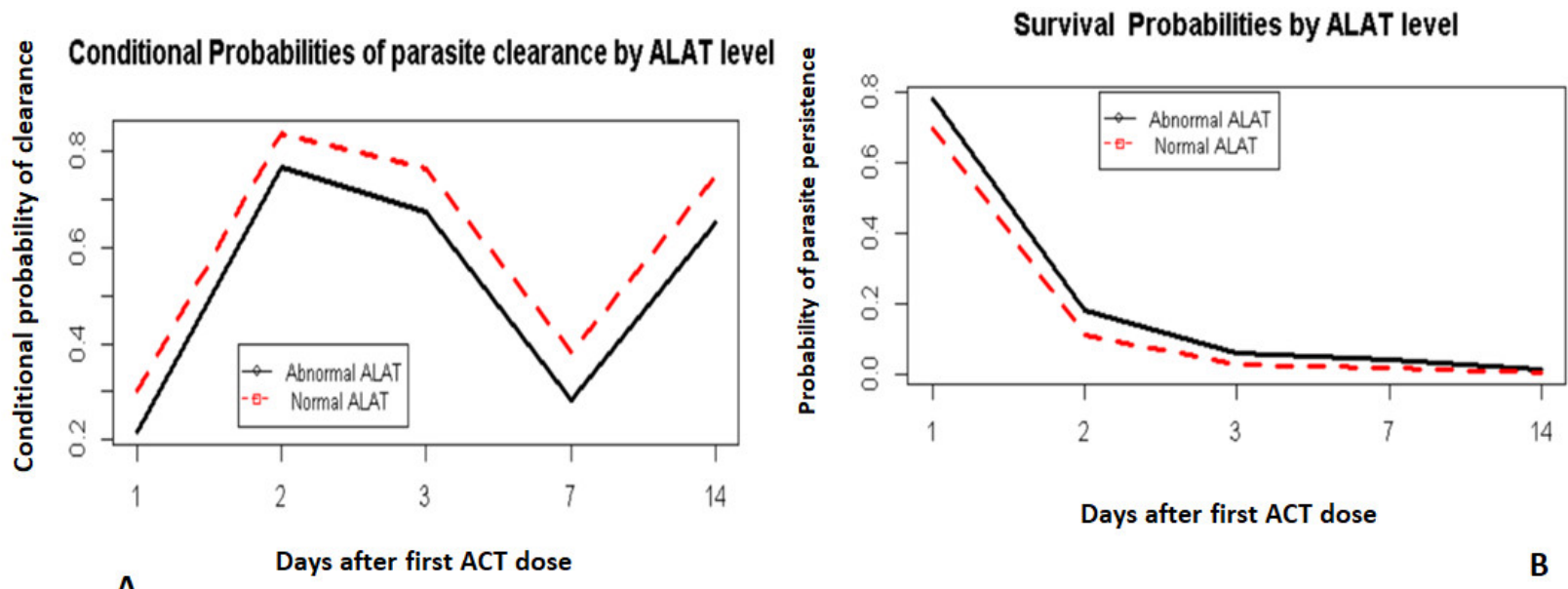

Figure 3. Hazard and survival probabilities by ALAT level. Conditional probabilities of parasite clearance by ALAT level (A). Survival probabilities by ALAT level (B).

Day 0 was not included. It corresponds to the day when the first dose of ACT was administered.

Lower time-to-clearance was also correlated in part with the participants' creatinine levels before treatment (Figure 4A,B). Those with a normal creatinine level (within normal limits) had a lower chance of clearing parasites by each visit day given they had not cleared in the previous day than those with abnormal creatinine levels (below the normal limits).
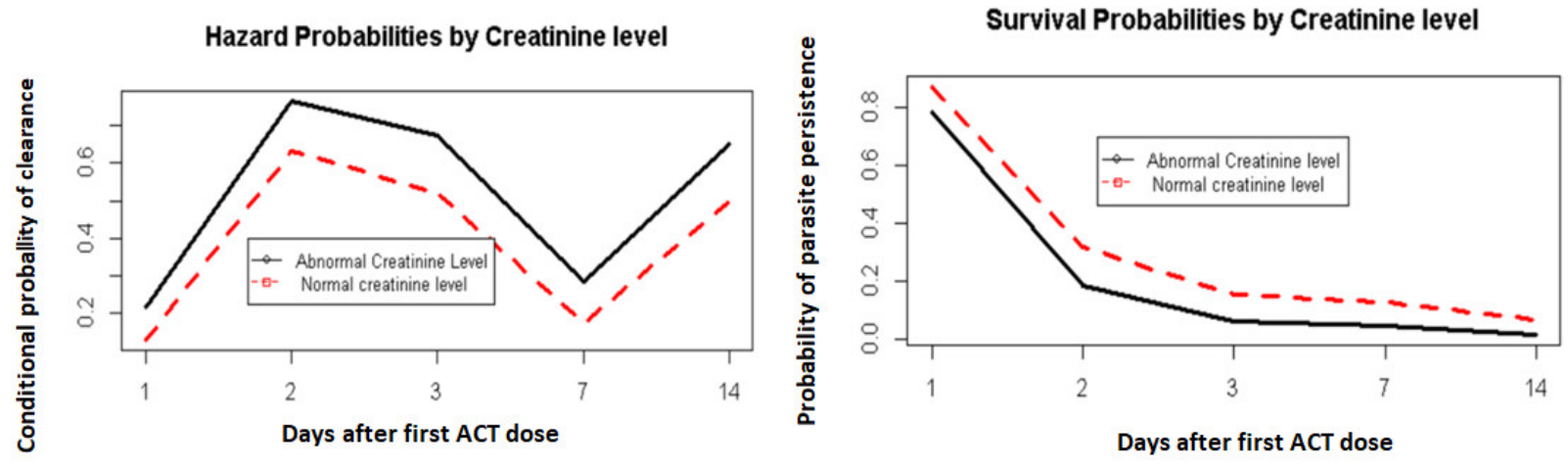

A

Figure 4. Hazard and survival probabilities by creatinine levels. Hazard probabilities by creatinine level (A). Survival probabilities by creatinine level (B).

Day 0 was not included. It corresponds to the day when the first dose of ACT was administered.

Although hemoglobin levels were not strongly correlated with parasite clearance, there was a trend in which those with lower pretreatment hemoglobin levels appeared to lag behind those with a normal pretreatment hemoglobin level (Figure 5A,B). This relationship was statistically insignificant $(p>0.05)$. 
Generally, there was no significant difference $(p>0.05)$ in the parasite clearance rates with time when comparing patients on the three test drug regimens.

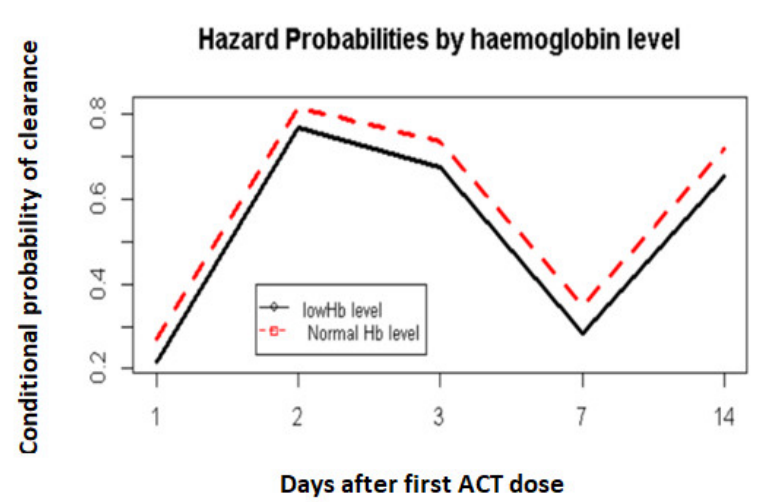

A

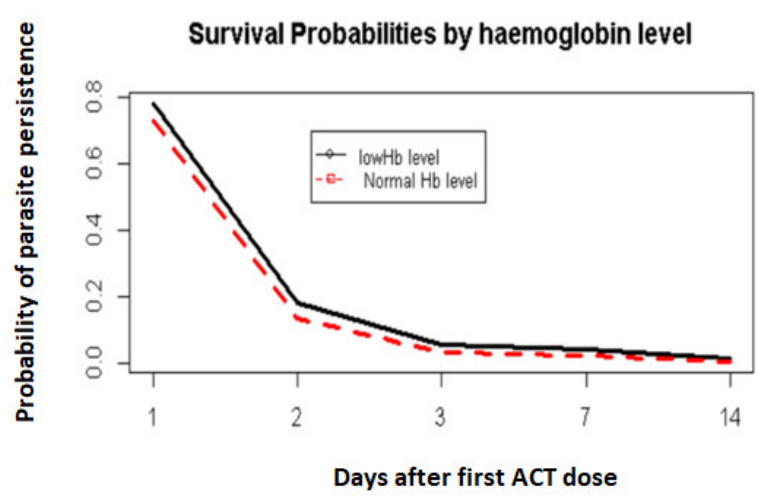

B

Figure 5. Hazard and survival probabilities by hemoglobin. Hazard probabilities by haemoglobin level (A). Survival probabilities by haemoglobin level (B).

Day 0 was not included. It corresponds to the day when the first dose of ACT was administered.

\section{Discussion}

This study aimed to model P. falciparum clearance among children aged 6 to 120 months in two ecologically different regions (Mutengene and Garoua) following treatment with AL, AS-AQ and DHA-PPQ. A discrete-time survival model was fitted to predict the factors in the two different transmission settings that may be correlated with parasite clearance during the early days of treatment.

There are many contributing and interrelated factors that may be associated with host response to parasite clearance [24]. Integrating these factors in models gives a better estimation of the extent to which each contributing factor is changing the rate of parasite clearance. In the present study, the interaction between age group and site was significantly associated with parasite clearance. In Mutengene, older children (60-120 months) had a higher probability of clearing the parasites when compared to the younger children (6-59 months). This observation is consistent with reports that immunity to malaria is age-dependent [25]. In a study that modeled factors associated with the half-life following treatment with $\mathrm{ACT}$, the authors observed that clearance of parasites irrespective of artemisinin treatment was related to immunoglobulins directed against erythrocyte surface ligands such as Plasmodium falciparum erythrocyte membrane protein 1 (PfEMP1) [26]. This effect was plasma-level-dependent and increased with age [26]. Contrarily, different studies carried out in Mozambique [27] and Malawi [28] identified the age of first exposure to P. falciparum as an insignificant predictor of antibody acquisition. In Garoua, a region with low humidity, it was observed that younger children appeared to clear parasites faster than older children. This observation is inconsistent with findings from an area with similar ecological endemicity and characteristics [26]. Our finding appeared to disagree with those of a study conducted in a low malaria transmission area in Mali that reported increase in age as a surrogate marker for immunity [26]. Previous studies have documented lower susceptibility to malaria infections in sympatric ethnic populations, and this was associated with higher malaria-specific immunoglobulin plasma levels, especially among the Fulani [29-31].

The model indicated that children in Mutengene lagged behind those in Garoua in clearing their parasites, especially during the first two days after drug administration. While the efficacies of the ACT were high and comparable [20], independent risk factors that accounted for persistent parasitemia on days 1 and 2 after drug administration included 
endemicity and age. An opposite age effect was seen between sites, which may be due to differences in chronologies in the development of malaria immunity. On the other hand, pretreatment hemoglobin level had a moderate effect on parasite persistence, although not significant at the 5\% significance level.

Patients with normal pretreatment serum ALAT activity and abnormal pretreatment serum creatinine levels appeared to clear their parasites faster than those with abnormal levels. Conversely, it was observed that children with normal creatinine levels cleared parasitemia more slowly after treatment. Abnormal ALAT activity and creatinine levels may be indicative of liver and kidney injuries, respectively. This may lead to compromises in the ability of patients to adequately maintain efficacious clearance patterns since increasingly resistant parasite forms may multiply in the presence of low drug concentrations. Self-medication is a common practice in most patients living in rural areas prior to hospital visit. The combined effect of the infection and self-medication could also increase the risk of not clearing parasites efficiently. Moreover, it was noticed that the rates of clearance between age groups and sites were not significantly different by day 3 . None of these parameters was associated with treatment failure during the study period, and efficacy levels remained high [20].

It has recently been shown through haplotype analysis of well-characterized clinical isolates of Plasmodium falciparum that genetic resistance to the artemisinins is mediated by polymorphisms in the gene encoding the Kelch propeller domain 13, and these mutations arise independently in several locations [32]. Studies conducted in Tanzania and Rwanda have also associated the nonsynonymous P. falciparum Kelch 13 mutations (P574L and $\mathrm{R} 561 \mathrm{H}$ ) with delayed parasite clearance [6-9]. The implication of these observations in close monitoring of resistant parasites cannot be overestimated especially in endemic areas where case management relies principally on the use of ACTs.

The major strength of this study is that it reported for the first time the factors influencing in vivo malaria parasite clearance in response to AS-AQ, AL and DHA-PPQ in two main transmission settings in Cameroon. The findings from this study are still valid today despite the fact that the clinical data were obtained from studies conducted between 2009 and 2013. This is because no recent data have shown a change in malaria transmission patterns in Mutengene and Garoua. Moreover, the ACTs are currently used in these areas in the treatment of uncomplicated P. falciparum malaria. Furthermore, results from this study add to baseline knowledge for ongoing investigations into regional differences in response to malaria interventions.

The limitations of this study are as follows: (1) The sample schedule for parasite density determination to estimate rates of parasite clearance was not optimal. Measurements using shorter time intervals are recommended by WHO in the early clearance phase after treatment initiation. With limited resources, the only operationally feasible strategy was to measure parasites on patient visit days. (2) Clearance times were estimated and not rates, with the latter more generally accepted to be a better measure of clearance. However, fitting parasite clearance in our discrete survival model provided an alternative way of effectively studying the behavior of parasites in the presence of drugs in sites of different malaria endemicities early during treatment. The plasma drug levels could not be measured to rule out reduced adherence or impairment in drug metabolism as causes of differences observed among study participants. Even though acetylation was used as a proxy of pharmacokinetics, it does not provide information on plasma drug levels. However, it is worth noting that the ethnic composition is different between the two sites despite the absence of data on ethnicity.

\section{Conclusions}

The model adopted for this study showed a delay in parasite clearance in Mutengene compared to Garoua among children aged 6 to 120 months treated with AS-AQ, DHAPPQ and AL. The site of study (Mutengene), increasing age (Mutengene) and normal pretreatment creatinine serum levels and normal pretreatment alanine aminotransferase 
serum levels were the main factors that significantly influenced parasite clearance in vivo after administration of ACTs. Although no differences were seen with parasite clearance on day 3 , the observations flag the need for close monitoring of host differential response to artemisinins in Cameroon.

Supplementary Materials: The following are available online at https: / www.mdpi.com/article/ 10.3390/pathogens10091106/s1, Supplementary Table S1. An example of data transformed to persontime data for fitting discrete-time survival model using logistic regression.

Author Contributions: Conceptualization, W.F.M., A.M.N. and I.M.A.; methodology, W.F.M., A.M.N. and I.M.A.; supervision, W.F.M. and G.F.; investigation, A.M.N., I.M.A., P.T.N.N., E.M.-S. and G.F.; data curation, A.M.N., I.M.A., P.T.N.N. and C.H.; software and formal analysis, A.M.N.; writing—original draft preparation, W.F.M., A.M.N. and I.M.A.; writing—review and editing, A.M.N., I.M.A. and P.T.N.N.; visualization, W.F.M., A.M.N., I.M.A., P.T.N.N. and G.F.; resource acquisition, W.F.M., A.M.N., I.M.A. and P.T.N.N. All authors have read and agreed to the published version of the manuscript.

Funding: Funding was received by W.F.M. from the WHO project number A60041 of the TDR/MIM program, the European Union Seventh Framework Programmes (FP7/2009-2014) under grant agreement No. 242095-EVIMalaR and from the University of Yaoundé I Cameroon-Pr \# UYI/FS/64.89/wfm. W.F.M., A.M.J., I.M.A. and P.T.N.N. are supported by the Malaria Research Capacity Development in West and Central Africa (MARCAD) Consortium through the Developing Excellence in Leadership, Training and Science (DELTAS) Africa Initiative (grant \# DEL-15-010) to the University of Yaoundé I. The DELTAS Africa Initiative is an independent funding scheme of the Alliance for Accelerating Excellence in Science in Africa (AESA) of the African Academy of Sciences (AAS) and supported by the New Partnership for Africa's Development Planning and Coordinating Agency (NEPAD Agency) with funding from the Wellcome Trust (grant \# 107741/A/15/Z) and the United Kingdom (UK) government. M.S.E. was supported by a US government grant to the University of Washington, Seattle, as a Fulbright Fellow. The funding agencies had no role in the design and conduct of the study.

Institutional Review Board Statement: The study was carried out following the rules of the Declaration of Helsinki of 1975 (https: / / www.wma.net/what-we-do/medical-ethics/declaration-ofhelsinki/ (accessed on 24 August 2021)), revised in 2013. The Institutional Review Boards of the Cameroon Baptist Health Services, the Biotechnology Center of the University of Yaoundé I and the WHO Ethical Review Committee approved the study. This clinical trial was registered at Www.clinicaltrials.gov (accessed on 24 August 2021) with registration number NCT01845701.

Informed Consent Statement: Written informed consent was obtained from guardians or parents before recruiting participants. Each parent/guardian was duly informed of the objectives, methods, anticipated benefits and potential hazards of the study. Consenting parents or guardians were also informed of their liberty to withdraw their children from the study at any time they wished without suffering any penalty.

Data Availability Statement: All relevant data are provided within the manuscripts. Raw data can be made available upon reasonable request.

Acknowledgments: The authors thank all those who accepted to take part in the study. Special thanks to the Cameroon Baptist Health Board for logistic support. We are indebted to the study participants, the study physicians and team for all their diligence during the study. The University of Yaoundé I, Cameroon, provided in-kind and administrative support.

Conflicts of Interest: The authors declare that they have no competing interest.

\section{Abbreviations}

ACT Artemisinin-based combination therapy

ALAT Alanine aminotransferase

AL Artemether-lumefantrine

AS-AQ Artesunate-amodiaquine

DHA-PPQ Dihydroartemisinin-piperaquine

WHO World Health Organization 


\section{References}

1. Barnes, K.I.; Durrheim, D.N.; Little, F.; Jackson, A.; Mehta, U.; Allen, E.; Dlamini, S.S.; Tsoka, J.; Bredenkamp, B.; Mthembu, D.J.; et al. Effect of artemether-lumefantrine policy and improved vector control on malaria burden in KwaZulu-Natal, South Africa. PLoS Med. 2005, 2, e330. [CrossRef]

2. Bhattarai, A.; Ali, A.S.; Kachur, S.P.; Mårtensson, A.; Abbas, A.K.; Khatib, R.; Al-Mafazy, A.-W.; Ramsan, M.; Rotllant, G.; Gerstenmaier, J.F.; et al. Impact of Artemisinin-Based Combination Therapy and Insecticide-Treated Nets on Malaria Burden in Zanzibar. PLoS Med. 2007, 4, e309. [CrossRef]

3. Wongsrichanalai, C.; Meshnick, S.R. Declining artesunate-mefloquine efficacy against falciparum malaria on the CambodiaThailand border. Emerg. Infect. Dis. 2008, 14, 716-719. [CrossRef] [PubMed]

4. Na-Bangchang, K.; Ruengweerayut, R.; Mahamad, P.; Ruengweerayut, K.; Chaijaroenkul, W. Declining in efficacy of a three-day combination regimen of mefloquine-artesunate in a multi-drug resistance area along the Thai-Myanmar border. Malar. J. 2010, 9, 273. [CrossRef] [PubMed]

5. Ariey, F.; Witkowski, B.; Amaratunga, C.; Beghain, J.; Langlois, A.-C.; Khim, N.; Kim, S.; Duru, V.; Bouchier, C.; Ma, L.; et al. A molecular marker of artemisinin-resistant Plasmodium falciparum malaria. Nature 2014, 505, 50-55. [CrossRef] [PubMed]

6. Tacoli, C.; Gai, P.P.; Bayingana, C.; Sifft, K.; Geus, D.; Ndoli, J.; Sendegeya, A.; Gahutu, J.B.; Mockenhaupt, F.P. Artemisinin resistance-associated K13 Polymorphisms of Plasmodium falciparum in Southern Rwanda, 2010-2015. Am. J. Trop. Med. Hyg. 2016, 95, 1090-1093. [CrossRef] [PubMed]

7. Bwire, G.M.; Ngasala, B.; Mikomangwa, W.P.; Kilonzi, M.; Kamuhabwa, A.A.R. Detection of mutations associated with artemisinin resistance at $\mathrm{k} 13$-propeller gene and a near complete return of chloroquine susceptible falciparum malaria in Southeast of Tanzania. Sci. Rep. 2020, 10, 3500. [CrossRef]

8. Uwimana, A.; Legrand, E.; Stokes, B.H.; Ndikumana, J.-L.M.; Warsame, M.; Umulisa, N.; Ngamije, D.; Munyaneza, T.; Mazarati, J.-B.; Munguti, K.; et al. Emergence and clonal expansion of in vitro artemisinin-resistant Plasmodium falciparum kelch13 R561H mutant parasites in Rwanda. Nat. Med. 2020, 26, 1602-1608. [CrossRef]

9. Uwimana, A.; Umulisa, N.; Venkatesan, M.; Svigel, S.S.; Zhou, Z.; Munyaneza, T.; Habimana, R.M.; Rucogoza, A.; Moriarty, L.F.; Sandford, R.; et al. Association of Plasmodium falciparum kelch13 R561H genotypes with delayed parasite clearance in Rwanda: An open-label, single-arm, multicentre, therapeutic efficacy study. Lancet Infect. Dis. 2021, 21, 1120-1128. [CrossRef]

10. Anderson, T.J.C.; Nair, S.; Nkhoma, S.; Williams, J.T.; Imwong, M.; Yi, P.; Socheat, D.; Das, D.; Chotivanich, K.; Day, N.P.J.; et al. High Heritability of Malaria Parasite Clearance Rate Indicates a Genetic Basis for Artemisinin Resistance in Western Cambodia. J. Infect. Dis. 2010, 201, 1326-1330. [CrossRef]

11. Boni, M.F.; Smith, D.; Laxminarayan, R. Benefits of using multiple first-line therapies against malaria. Proc. Natl. Acad. Sci. USA 2008, 105, 14216-14221. [CrossRef] [PubMed]

12. Petersen, I.; Eastman, R.; Lanzer, M. Drug-resistant malaria: Molecular mechanisms and implications for public health. FEBS Lett. 2011, 585, 1551-1562. [CrossRef]

13. Francis, D.; Nsobya, S.L.; Talisuna, A.; Yeka, A.; Kamya, M.R.; Machekano, R.; Dokomajilar, C.; Rosenthal, P.J.; Dorsey, G. Geographic differences in antimalarial drug efficacy in uganda are explained by differences in endemicity and not by known molecular markers of drug resistance. J. Infect. Dis. 2006, 193, 978-986. [CrossRef] [PubMed]

14. Greenhouse, B.; Slater, M.; Njama-Meya, D.; Nzarubara, B.; Maiteki-Sebuguzi, C.; Clark, T.D.; Staedke, S.G.; Kamya, M.R.; Hubbard, A.; Rosenthal, P.J.; et al. Decreasing efficacy of antimalarial combination therapy in Uganda is explained by decreasing host immunity rather than increasing drug resistance. J. Infect. Dis. 2009, 199, 758-765. [CrossRef] [PubMed]

15. Smith, D.L.; Klein, E.Y.; McKenzie, F.E.; Laxminarayan, R. Prospective strategies to delay the evolution of anti-malarial drug resistance: Weighing the uncertainty. Malar. J. 2010, 9, 217. [CrossRef]

16. Maude, R.J.; Pontavornpinyo, W.; Saralamba, S.; Aguas, R.; Yeung, S.; Dondorp, A.M.; Day, N.P.J.; White, N.J.; White, L.J. The last man standing is the most resistant: Eliminating artemisinin-resistant malaria in Cambodia. Malar. J. 2009, 8, 31. [CrossRef]

17. Okell, L.C.; Drakeley, C.J.; Bousema, T.; Whitty, C.; Ghani, A. Modelling the Impact of Artemisinin Combination Therapy and Long-Acting Treatments on Malaria Transmission Intensity. PLoS Med. 2008, 5, e226. [CrossRef]

18. Pongtavornpinyo, W.; Yeung, S.; Hastings, I.M.; Dondorp, A.M.; Day, N.P.; White, N.J. Spread of anti-malarial drug resistance: Mathematical model with implications for ACT drug policies. Malar. J. 2008, 7, 229. [CrossRef]

19. Sayang, C.; Gausseres, M.; Vernazza-Licht, N.; Malvy, D.; Bley, D.; Millet, P. Treatment of malaria from monotherapy to artemisinin-based combination therapy by health professionals in urban health facilities in Yaoundé, central province, Cameroon. Malar. J. 2009, 8, 176. [CrossRef]

20. Nji, A.M.; Ali, I.M.; Moyeh, M.N.; Ngongang, E.-O.; Ekollo, A.M.; Chedjou, J.-P.; Ndikum, V.N.; Evehe, M.S.; Froeschl, G.; Heumann, C.; et al. Randomized non-inferiority and safety trial of dihydroartemisin-piperaquine and artesunate-amodiaquine versus artemether-lumefantrine in the treatment of uncomplicated Plasmodium falciparum malaria in Cameroonian children. Malar. J. 2015, 14, 27. [CrossRef]

21. World Health Organization. Methods for Surveillance of Antimalarial Drug Efficacy; World Health Organization: Geneva, Switzerland, 2009

22. World Health Organization. UNICEF/UNDP/World Bank/WHO Special programme for research and training in tropical diseases. In Microscopy for the Detection, Identification and Quantification of Malaria Parasites on Stained Thick and Thin Blood Films in Research Settings (Version 1.0): Procedure: Methods Manual; World Health Organization: Geneva, Switzerland, 2015. 
23. Singer, J.D.; Willett, J.B. It's About Time: Using discrete-time survival analysis to study duration and the timing of events. J. Educ. Stat. 1993, 18, 155-195.

24. White, N.J. Malaria parasite clearance. Malar. J. 2017, 16, 88. [CrossRef]

25. Rodriguez-Barraquer, I.; Arinaitwe, E.; Jagannathan, P.; Kamya, M.R.; Rosenthal, P.J.; Rek, J.; Dorsey, G.; Nankabirwa, J.; Staedke, S.G.; Kilama, M.; et al. Quantification of anti-parasite and anti-disease immunity to malaria as a function of age and exposure. Elife 2018, 7, e35832. [CrossRef]

26. Lopera-Mesa, T.M.; Doumbia, S.; Chiang, S.; Zeituni, A.E.; Konate, D.S.; Doumbouya, M.; Keita, A.S.; Stepniewska, K.; Traore, K.; Diakite, S.A.S.; et al. Plasmodium falciparum Clearance Rates in Response to Artesunate in Malian Children with Malaria: Effect of Acquired Immunity. J. Infect. Dis. 2013, 207, 1655-1663. [CrossRef]

27. Nhabomba, A.J.; Guinovart, C.; Jiménez, A.; Manaca, M.N.; Quintó, L.; Cisteró, P.; Aguilar, R.; Barbosa, A.; Rodríguez, M.H.; Bassat, Q.; et al. Impact of age of first exposure to Plasmodium falciparum on antibody responses to malaria in children: A randomized, controlled trial in Mozambique. Malar. J. 2014, 13, 121. [CrossRef]

28. Barua, P.; Beeson, J.G.; Maleta, K.; Ashorn, P.; Rogerson, S.J. The impact of early life exposure to Plasmodium falciparum on the development of naturally acquired immunity to malaria in young Malawian children. Malar. J. 2019, 18, 11. [CrossRef]

29. Bolad, A.; Farouk, S.E.; Israelsson, E.; Dolo, A.; Doumbo, O.K.; Nebie, I.; Maiga, B.; Kouriba, B.; Luoni, G.; Sirima, B.S.; et al. Distinct Interethnic Differences in immunoglobulin G class/subclass and immunoglobulin m antibody responses to malaria antigens but not in immunoglobulin g responses to non-malarial antigens in sympatric tribes living in West Africa. Scand. J. Immunol. 2005, 61, 380-386. [CrossRef]

30. Flegg, J.A.; Guérin, P.J.; Nosten, F.; Ashley, E.A.; Phyo, A.P.; Dondorp, A.M.; Fairhurst, R.M.; Socheat, D.; Borrmann, S.; Björkman, A.; et al. Optimal sampling designs for estimation of Plasmodium falciparum clearance rates in patients treated with artemisinin derivatives. Malar. J. 2013, 12, 411. [CrossRef]

31. Nkhoma, S.C.; Stepniewska, K.; Nair, S.; Phyo, A.P.; McGready, R.; Nosten, F.; Anderson, T.J.C. Genetic evaluation of the performance of malaria parasite clearance rate metrics. J. Infect. Dis. 2013, 208, 346-350. [CrossRef]

32. Takala-Harrison, S.; Jacob, C.G.; Arze, C.; Cummings, M.P.; Silva, J.C.; Dondorp, A.M.; Fukuda, M.M.; Hien, T.T.; Mayxay, M.; Noedl, H.; et al. Independent emergence of artemisinin resistance mutations among Plasmodium falciparum in Southeast Asia. J. Infect. Dis. 2015, 211, 670-679. [CrossRef] [PubMed] 